\title{
Descriptive Analysis of a Telephone Based Community Monitoring Service for COVID-19
}

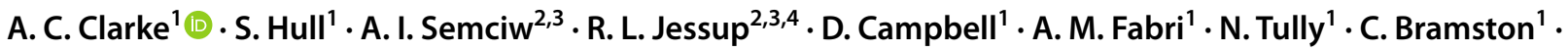 \\ J. Hayes ${ }^{1}$
}

Accepted: 28 April 2021 / Published online: 11 May 2021

(c) The Author(s), under exclusive licence to Springer Science+Business Media, LLC, part of Springer Nature 2021

\begin{abstract}
The coronavirus disease (COVID-19) pandemic has required health services to rapidly respond to the needs of people diagnosed with the virus. Over $80 \%$ of people diagnosed with COVID-19 experience a mild illness and there is a need for community management to support these people in their home. In this paper we present, a telephone based COVID-19 community monitoring service developed in an Australian public health network, and we describe the rapid implementation of the service and the demographic and clinical characteristics of those enrolled. A retrospective mixed methods evaluation of the COVID-19 community monitoring service using the RE-AIM (Reach, Effectiveness, Adoption, Implementation, and Maintenance) framework. Eight hundred and fifty COVID-19 positive patients were enrolled, $54 \%$ female, $45 \%$ male, mean age 34 years SD 17. Four hundred and nine (48\%) patients were born outside Australia. Among the 850 patients, 305 (36\%) were classified as having a high risk of serious illness from COVID-19. The most prevalent risk factors were cardiovascular disease (37\%), lung disease (30\%) and age over 60 years (26\%). The most common reported ongoing symptoms were fatigue (55\%), breathing issues (26\%) and mental health issues such as low mood (19\%). There were no deaths in patients that participated in the service. The process of risk stratification undertaken with telephone triage was effective in determining risk of prolonged illness from COVID-19. Telephone monitoring by trained health professionals has a strong potential in the effective management of patients with a mild COVID-19 illness.
\end{abstract}

Keywords Telemedicine $\cdot$ Community medicine $\cdot$ Community health services

\section{Introduction}

The coronavirus disease (COVID-19) pandemic has required health services to rapidly respond to the needs of people diagnosed with the virus. The spectrum of disease has been categorised as mild, severe or critical [1]. Early findings from China established that $81 \%$ of people with COVID19 had mild disease, $14 \%$ had severe disease and 5\% had

A. C. Clarke

Andrea.clarke4@nh.org.au

1 Hospital Without Walls Directorate, Northern Health, 1231 Plenty Road, Bundoora, Melbourne, VIC 3083, Australia

2 Allied Health Research, Northern Health, Melbourne, Australia

3 School of Allied Health, Human Services and Sport, La Trobe University, Melbourne, Australia

4 School of Rural Health, Monash University, Warragul, Australia critical disease; fatality rates were reported to be $49 \%$ in those with critical disease [2]. Individuals that are older or have co-morbidities such as cardiovascular disease, chronic lung disease, diabetes and obesity are at high risk of critical illness from COVID-19 [1].

Over $80 \%$ of people with COVID-19 are likely to experience a mild illness [1]. There is a need for community management of those with mild illness to reduce the strain on hospital resources and healthcare worker exposure [3, 4]. Community management involves monitoring symptoms while individuals remain at home, while also allowing for timely identification of deteriorating symptoms which usually occurs around one week after illness onset $[3,5]$.

Due to the novel nature of this coronavirus, there is limited evidence on the most successful healthcare model for community management of COVID-19 patients. Community management models described in the literature to date generally utilised a combination of monitoring technology and telephone support from health professionals. Kricke et al. 
[6]; Morgan et al. [7] and Medina et al. [8] all described the implementation of an electronic Short Message Service (SMS) monitoring system that relied on patient response of self-reported symptoms, with phone calls made by health professionals only to those patients with self-reported symptoms that were of concern. Silven et al. [3] described a model that combined monitoring of vital signs with video consultations. There appears to be an absence of research on lower technology models of care.

In this study, we describe the development and outcomes of a telephone based community monitoring service for patients with COVID-19. The aim of this study was to describe the rapid implementation of a telephone based COVID-19 community monitoring service and the demographic and clinical characteristics of those enrolled.

\section{Methods}

\section{Design}

This study was a retrospective mixed methods evaluation of the COVID-19 community monitoring service using the RE-AIM (Reach, Effectiveness, Adoption, Implementation, and Maintenance) framework [9].

\section{Setting}

The World Health Organization (WHO) declared a international public health emergency on the 30th January 2020, around the same time Australia recorded its first confirmed case of COVID-19. Australia's largest COVID-19 outbreak was in Melbourne, Victoria, with $72 \%$ (20,345 on 1st December 2020) [10] of the nation's cases recorded in Victoria and 67\% $(n=18,965$ on 1 st December 2020) [11] in metropolitan Melbourne. The new daily cases in Victoria began to rise in late June 2020 and peaked on 5th August 2020 with 725 new cases reported in a $24 \mathrm{~h}$ period [12].

Northern Health is the key provider of public health care in Melbourne's northern suburbs. Residents in the Northern Health catchment are culturally and linguistically diverse, born in over 170 countries, speaking over 100 different languages and following 75 religions or beliefs [13]. Northern Health's catchment accounts for approximately $10 \%$ of Victoria's population, however at the peak of the pandemic, one third of Victoria's COVID-19 cases resided in the catchment.

\section{Participants}

All patients that consented to participate in the COVID-19 community monitoring service (the service) were included in the evaluation. Patients were enrolled in the service if they were diagnosed with COVID-19 and were a patient of
Northern Health and/or lived in the Northern Health catchment area. Patients referred were contacted by the service, after the patient had been notified of a positive COVID-19 result.

\section{Procedure}

The service was established to ensure that people who were COVID-19 positive living in Melbourne's northern region were provided with health advice and support during the acute phase of their infection. The service also aimed to provide timely identification of worsening symptoms and prevent unnecessary hospital admissions.

A low technology model was rapidly developed that relied on telephone contact by trained health professionals. The development of the service was first discussed on 15th July 2020 and the service went live on 21 st July 2020. Nursing staff furloughed due to medical risk issues and allied health staff from community programs were engaged to work in the service. The service was suspended on the 12th November 2020 due to there no longer being any active cases in the state.

Patients were monitored by the service during the acute phase of their illness which was typically up to 14 days. All patients received an initial call within $24-48 \mathrm{~h}$ of receipt of referral. At the initial call, consent was gained for the patient to be monitored by the service, and a clinical assessment incorporating risk stratification and a social and welfare needs assessment were undertaken. Education was provided on how to isolate at home and how to identify symptoms of deterioration.

A telephone call script was used during the initial and subsequent calls. The initial call script included a checklist of risk factors and both the initial and subsequent call scripts included a checklist of symptoms. The telephone scripts were refined through iterative processes during the first four weeks of the service. The risk factor checklist supported the assessment of risk of serious illness from COVID-19, and risk stratified the patient as high or low risk (see Table 1). The symptom checklist identified if patient symptoms were worsening. Patient symptoms were categorised as stable or deteriorating. For patients with deteriorating symptoms, escalation of care involved either consultation with a Northern Health medical officer, referral to the general practitioner or hospital emergency department (ED), or calling an ambulance. High risk patients received daily monitoring calls and low risk patients received second daily contact. (See Fig. 1 for more information).

Patients were discharged from the service when cleared from isolation by the Victorian Department of Health and Human Services (DHHS). At the time the service was active, the DHHS were responsible for contact tracing and release from isolation. All patients received a post discharge follow 
Table 1 Risk factor stratification criteria

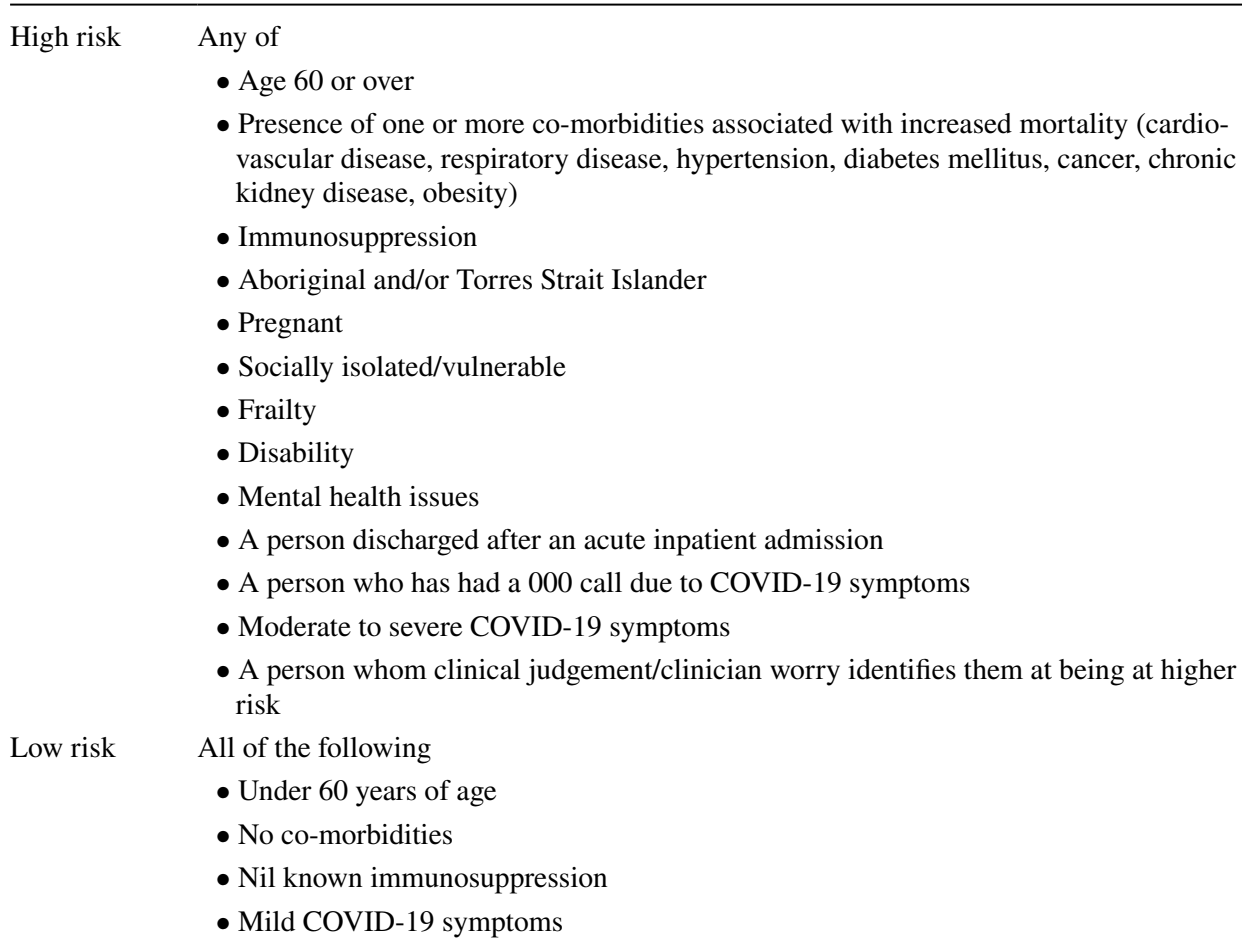

- Presence of one or more co-morbidities associated with increased mortality (cardiovascular disease, respiratory disease, hypertension, diabetes mellitus, cancer, chronic kidney disease, obesity)

- Immunosuppression

- Aboriginal and/or Torres Strait Islander

- Pregnant

- Socially isolated/vulnerable

- Frailty

- Disability

- Mental health issues

- A person discharged after an acute inpatient admission

- A person who has had a 000 call due to COVID-19 symptoms

- Moderate to severe COVID-19 symptoms

- A person whom clinical judgement/clinician worry identifies them at being at higher risk

Low risk All of the following

- Under 60 years of age

- No co-morbidities

- Nil known immunosuppression

- Mild COVID-19 symptoms

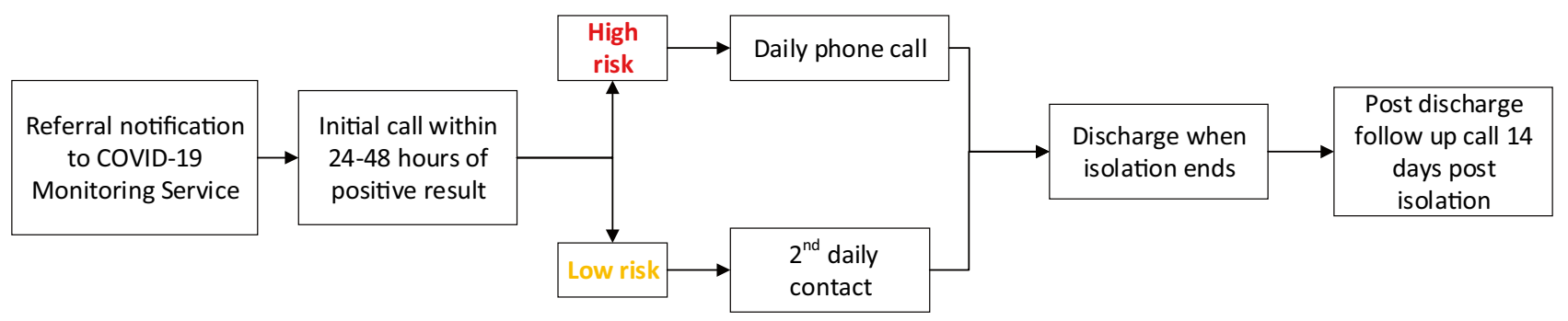

Fig. 1 Patient flow

up call two weeks after discharge to check on their recovery, self-reported ongoing symptoms and if they had returned to their usual daily activities.

\section{Evaluation Procedure}

Patient data (demographic) was extracted from the Northern Health data warehouse. Socio-economic Indexes for Areas (SEIFA) 2016, ranking data was obtained from the Australian Bureau of Statistics (ABS) and the residential suburb data was used to determine decile rankings for the state of Victoria. Deciles are ordered from lowest to highest with decile 1 being the most disadvantaged and 10 being the least.

Data on patient demographics, risk level, self-reported symptoms and issues during acute illness, clinical recommendations and symptoms or issues beyond 30 days after symptom onset was gathered from an audit of the clinical progress notes.

\section{Data Analysis}

\section{Descriptive Analysis of Service Utilization}

Demographics were described based on age, country of birth, preferred language, SEIFA ranking and gender. SEIFA was dichotomized into high, low, with those $\leq$ being classified as low. Gender has been reported as male, female and other. Due to the low reporting of 'other' within our sample, any further analyses including gender as a variable have been conducted with male and female levels of sex only (see Fig. 2 for patient age and gender distribution). The proportion of people who satisfied each risk category was also described. 


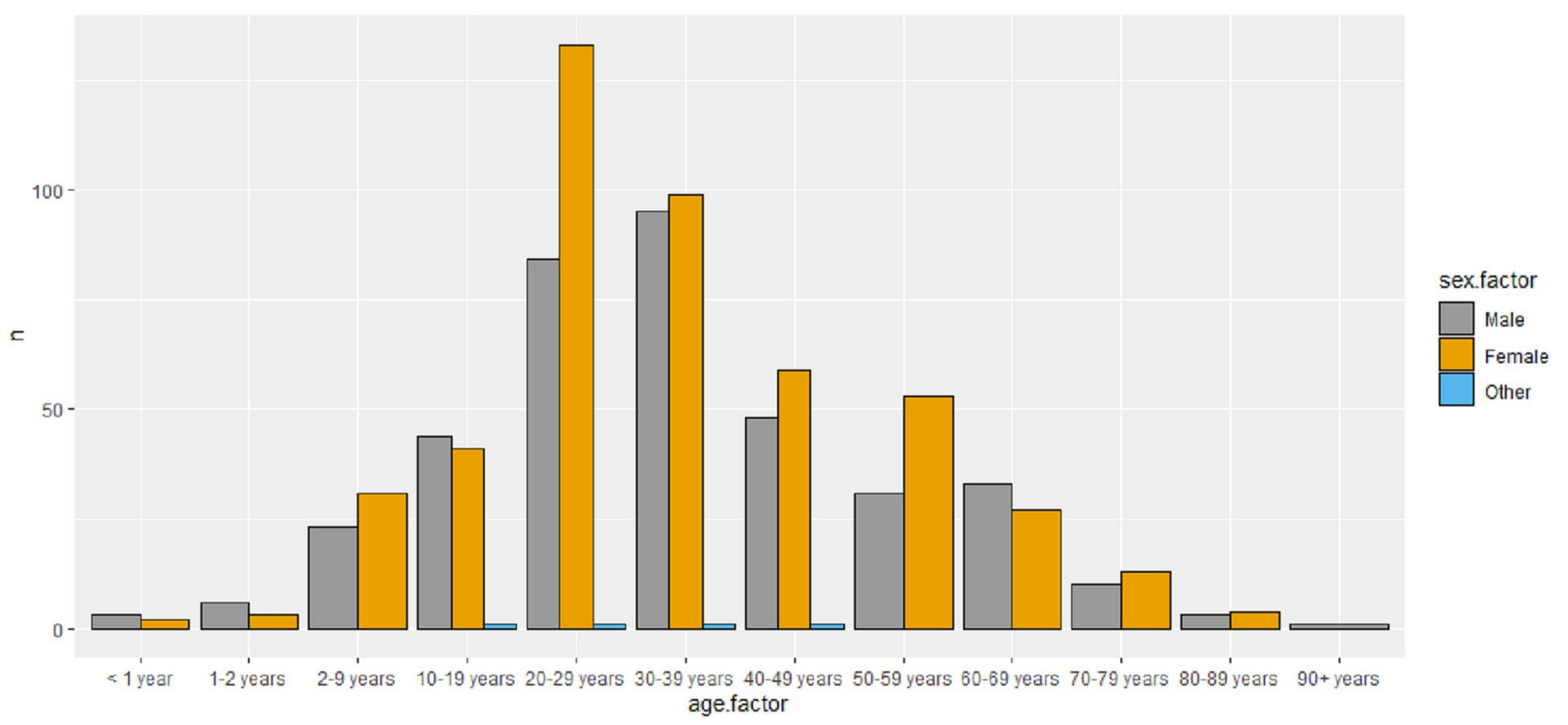

Fig. 2 Patient age and gender distribution

\section{Factors Associated with Ongoing Symptoms}

Only those who agreed to receive post discharge follow-up calls, or who responded to follow-up calls were included in this analysis. Factors associated with ongoing symptoms were investigated using multivariable logistic regression. The outcome variable was 'ongoing symptoms' (yes/no), and the independent variables were 'risk category' (high/ low), gender (male/female), SEIFA (high/low). Language/ use of interpreter services and country of birth were not included due to their potential relationship with SEIFA (collinearity). Age was not included, as it was a variable used to determine risk category. Results are presented as crude and adjusted odds ratios (95\% CI). All analyses were performed using R statistical software (https://www.r-project.org/, version 4.0.2).

\section{Results}

\section{Characteristics of the Patients}

Between 21st July and 12th November 2020, 850 COVID19 positive patients were enrolled, $54 \%$ female, $45 \%$ male, mean age (SD) 34 (17) years. Four hundred and nine (48\%) patients were born outside Australia. Three hundred and five (36\%) were classified as high risk of serious illness from COVID-19. The most prevalent risk factors were cardiovascular disease $(n=112,37 \%)$, lung disease $(n=92,30 \%)$, aged over 60 years $(n=80,26 \%)$ and diabetes $(n=60$, $20 \%)$. There were no deaths in patients that participated in the service. Thirty (3.5\%) patients had deteriorating symptoms and were escalated for urgent medical care. Forty-one patients presented to the emergency department, 22 patients were admitted to hospital and one required an ICU admission. Characteristics of the patients are shown in Table 2.

\section{Prolonged IIIness}

Prolonged illness data is available from the 646 patients that responded to or accepted follow-up calls, representing $76 \%$ of the total sample. Of those that responded to the follow up calls 415 were categorized as low risk and 231 high risk. Of the 646 patients, $16 \%(n=105)$ patients reported ongoing symptoms 20 days post illness onset. The most common reported ongoing symptoms were fatigue $(n=58 ; 55 \%)$, breathing issues $(n=27 ; 26 \%)$ and mental health issues such as low $\operatorname{mood}(\mathrm{n}=20 ; 19 \%)$ (see Fig. 3$)$.

Crude analyses suggested that those deemed high risk had $2.18(1.42,3.33, \mathrm{p}<0.001)$ times greater odds of prolonged symptoms than those deemed low risk. Sex [odds ratio $(O R)=1.52(0.98,2.34), p=0.073]$ or SEIFA category $[\mathrm{OR}=1.12(0.71,1.77), \mathrm{p}=0.753]$ were not associated with prolonged symptoms. Figure 4 illustrates a forest plot of the relationship between risk category and the odds of prolonged symptoms, after adjusting for sex and SEIFA category. Adjustments did not change the meaning of the outcome. Adjusted probabilities of prolonged symptoms in those deemed high and low risk were $23 \%(18 \%, 29 \%)$ and $12 \%(9 \%, 16 \%)$ respectively. There were no interactions between risk category and sex $(\mathrm{p}=0.98)$, or risk and SEIFA category $(\mathrm{p}=0.48)$ (see Fig. 4$)$. 
Table 2 Characteristics of enrolled patients

\begin{tabular}{|c|c|}
\hline Total, $\mathrm{n}$ & 850 \\
\hline Age, mean (SD) & $34(17)$ \\
\hline Female, n (\%) & $460(54.1)$ \\
\hline Male, $\mathrm{n}(\%)$ & $384(45.1)$ \\
\hline Other, $\mathrm{n}(\%)$ & $6(0.7)$ \\
\hline \multicolumn{2}{|l|}{ Country of birth (top 8), n (\%) } \\
\hline Australia & $436(51.2)$ \\
\hline India & $109(12.8)$ \\
\hline Iraq & $59(6.9)$ \\
\hline Nepal & $40(4.7)$ \\
\hline New Zealand & $16(1.8)$ \\
\hline Philippines & $24(2.8)$ \\
\hline Samoa & $17(2)$ \\
\hline Sri lanka & $13(1.5)$ \\
\hline \multicolumn{2}{|l|}{ Preferred language (top 4), n (\%) } \\
\hline English & $628(73.8)$ \\
\hline Arabic & $27(3.1)$ \\
\hline Assyrian & $15(1.7)$ \\
\hline Chaldean & $9(1)$ \\
\hline \multicolumn{2}{|l|}{ SEIFA ranking within state, decile, $\mathrm{n}(\%)$} \\
\hline 1 & $177(20)$ \\
\hline 2 & $69(8)$ \\
\hline 3 & $292(34)$ \\
\hline 4 & $8(0.9)$ \\
\hline 5 & $35(4)$ \\
\hline 6 & $80(9)$ \\
\hline 7 & $49(6)$ \\
\hline 8 & $99(11)$ \\
\hline 9 & $31(3)$ \\
\hline 10 & $10(1)$ \\
\hline High risk, $\mathrm{n}(\%)$ & $302(35)$ \\
\hline \multicolumn{2}{|l|}{ Risk factors, $\mathrm{n}(\%)^{\mathrm{a}}$} \\
\hline Patients with $>1$ risk factor & $192(64)$ \\
\hline Aged 60 or over & $80(26)$ \\
\hline Aboriginal and/or Torres Strait Islander & $6(2)$ \\
\hline Lung disease & $92(30)$ \\
\hline Cardiovascular disease & $112(37)$ \\
\hline Diabetes & $60(20)$ \\
\hline Pregnancy & $17(6)$ \\
\hline Socially isolated/vulnerable & 13 \\
\hline Disability & $24(8)$ \\
\hline Frailty & $12(4)$ \\
\hline Cancer & $12(4)$ \\
\hline Chronic renal disease & $15(5)$ \\
\hline Obesity & $52(17)$ \\
\hline Immunosuppressed & $22(7)$ \\
\hline Inpatient admission for COVID-19 & $64(21)$ \\
\hline 000 call due to COVID & $11(4)$ \\
\hline Moderate to severe symptoms & $9(3)$ \\
\hline Clinician worry & $4(1)$ \\
\hline Escalation of medical care, $\mathrm{n}(\%)$ & $30(3.5)$ \\
\hline Hospital admission, n (\%) & $22(3.7)$ \\
\hline Length of hospital admission stay, mean days, $\mathrm{SD}^{\mathrm{b}}$ & $4.8(4.7)$ \\
\hline ED presentation & $41(4.8)$ \\
\hline ICU admission, $\mathrm{n}(\%)$ & $1(0.1)$ \\
\hline
\end{tabular}

Table 2 (continued)

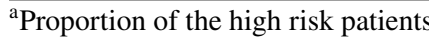

${ }^{\mathrm{b}}$ Applies only to those patients that were admitted to hospital

\section{Discussion}

This study examined the demographic and clinical characteristics of patients enrolled in a COVID-19 community monitoring service. One in five people who were categorised as high risk at the time of diagnosis went on to develop prolonged symptoms. Those at high risk were also twice as likely to experience prolonged symptoms than those categorised as low risk. Escalations for medical care, emergency department presentations and hospital admissions were low and there were no deaths in patients enrolled in the service. There was a high proportion of enrolled patients from lower socioeconomic and migrant backgrounds.

We found that $16 \%$ of enrolled patients who responded to follow up calls self-reported symptoms beyond 30 days from illness onset. This is consistent with findings reported in the literature. Greenhalgh et al. [14] reported approximately $10 \%$ of patients are likely to experience symptoms beyond three weeks and Medina et al. [8] found 10\% of patients required continued monitoring beyond 14 days due to persistent symptoms. Tenforde et al.'s [15] research reported that $65 \%$ of people had not returned to their usual state of health 14-21 days post illness onset.

This evaluation found that fatigue was the most common ongoing symptom affecting more than half of the patients with ongoing symptoms. This is less than reported in the studies by Eythorsson et al. [16], who reported ongoing lethargy in $74.7 \%$, and Tenforde et al. [15], who reported ongoing fatigue in $71 \%$ of patients. This may be attributed to the differing time points used in reporting of prolonged symptoms. Similar studies reported symptoms between 14 and 21 days $[15,16]$, whereas our study reported prolonged symptoms at least 30 days post illness, providing a greater period of time within which symptoms may have resolved.

In this evaluation, we found that $35 \%$ of patients were at high risk, and of those, $64 \%$ had more than one risk factor. This is higher than reported in the study by Medina et al. [8], who found $55 \%$ of patients had greater than one risk factor. Health risk factors were comparable between the studies, however socioeconomic and migrant backgrounds were not reported in other studies and may account for the differences seen between studies [8]. Only $3.5 \%$ of patients were escalated for urgent medical care which is lower than the research by Morgan et al. [7] who reported 15.5\% escalation for urgent assessment. This evaluation found that 41 patients presented to the ED and 22 were admitted. The proportion of hospitalised cases is well below Australia's national average of $13 \%$ (on 22 November 2020) [17]. The 
Fig. 3 Symptom type in those that presented with prolonged symptoms (total sample $=105)$
Prolonged symptoms

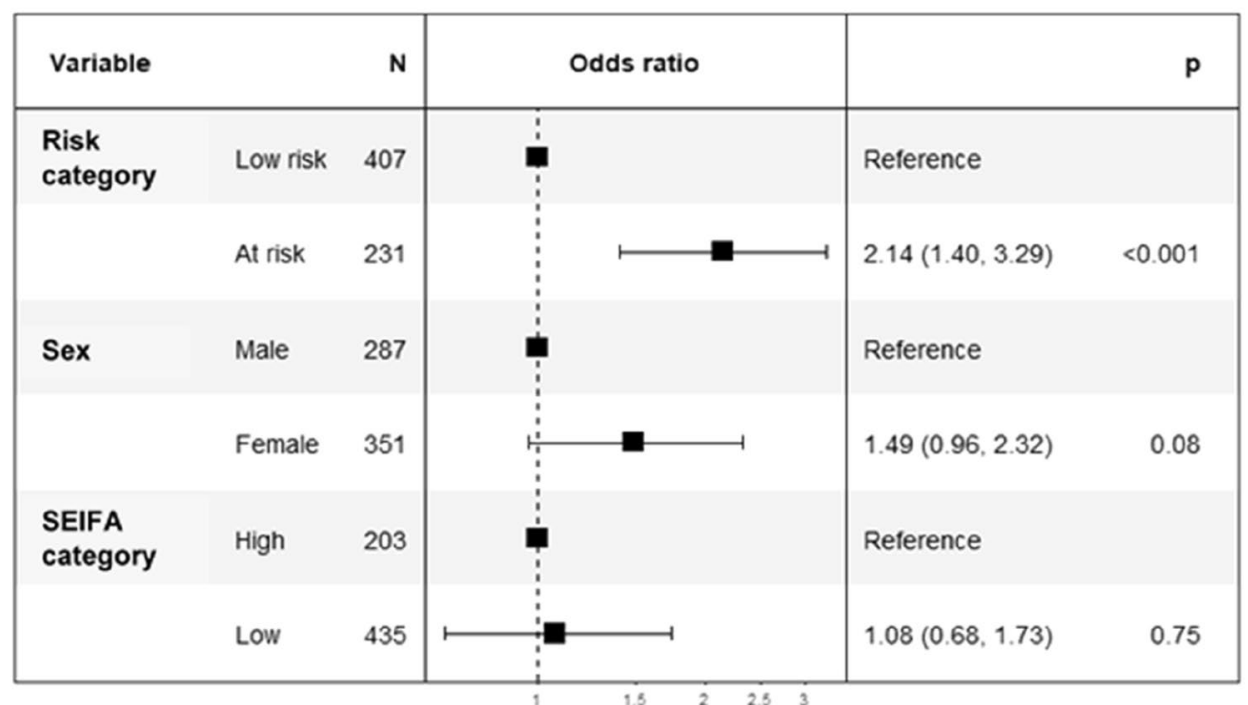

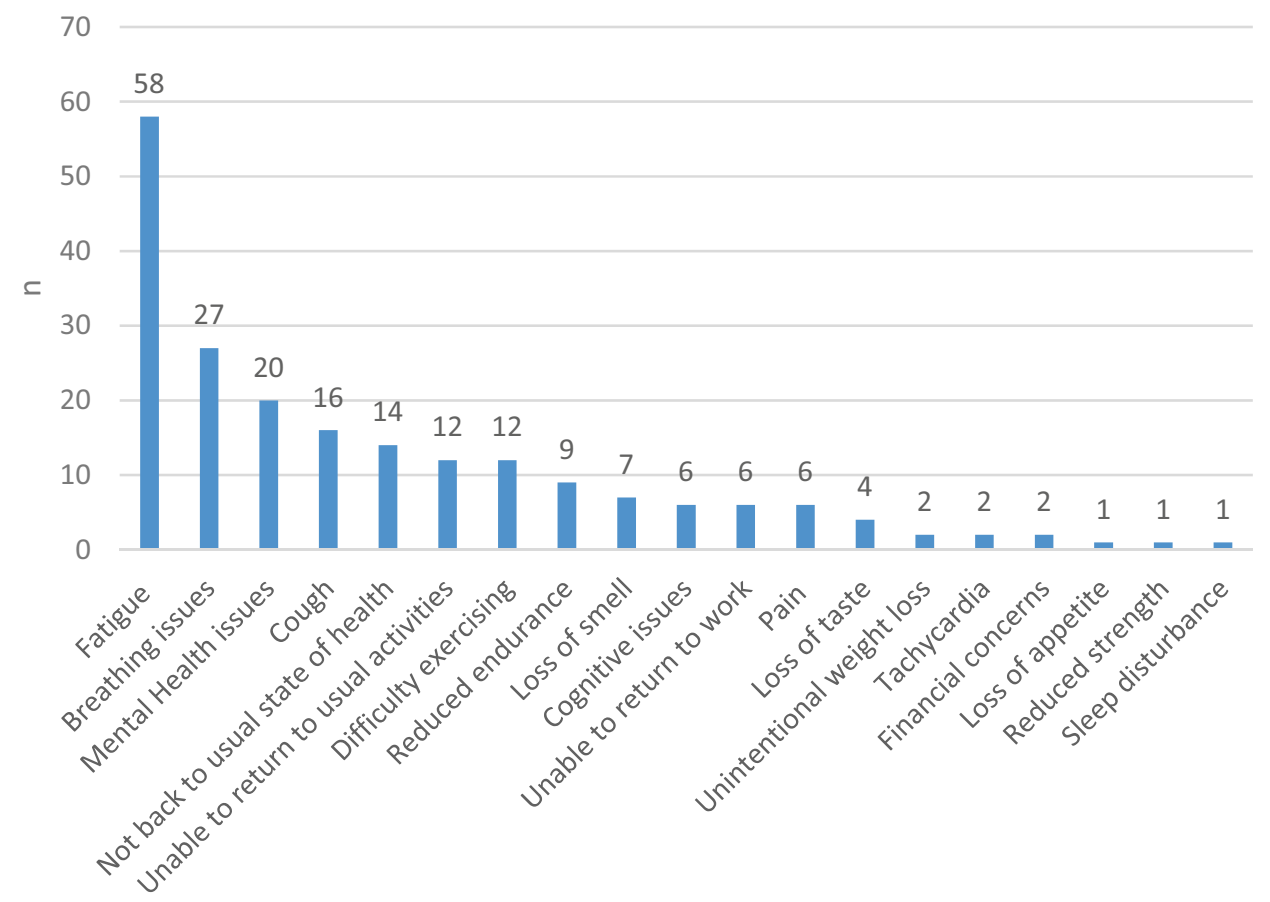

Fig. 4 Relationship between risk category, sex, Socioeconomic Indexes for Australia (SEIFA) and prolonged illness 
suggests that this low technology model is capable of monitoring and supporting people during the acute phase of COVID-19. Further research is required to determine if this model of care could also be suitable for monitoring people at home with other infectious diseases.

A limitation of the study is that findings are from a single health network and there was no comparator group so the findings may not be generalisable. There was also a need to commence the program rapidly which limited our ability to create a comparator group. As the program was rapidly developed, the evaluation was completed in retrospect and patient related outcome measures were unable to be included. Further research is required to determine the impact of community management on patient related outcomes such as quality of life.

\section{Conclusion}

This study described a telephone monitoring service for patients in the community with COVID-19. The service approach was a low technology model that relied on telephone monitoring from trained health professionals. One in five people identified as being high risk developed prolonged symptoms, and this was two times greater odds than those at low risk. The study showed that the telephone triage of risk stratification was effective in determining risk of prolonged illness from COVID-19. Telephone monitoring by trained health professionals has a strong potential in the effective management of patients with mild COVID-19 illness.

Author Contributions All authors participated in the design of the study. ACC, SH and NT participated in the data collection. ACC and AS participated in the data analysis and interpretation. ACC wrote the draft paper and all authors participated in editing it.

Funding This research received no external funding.

\section{Declarations}

Conflict of interest Authors have no conflicts of interest to declare.

Ethical Approval Northern Health Human Research Low Risk Ethics Committee (Reference 68253).

Consent to Participate Verbal consent was obtained from patients to participate in the service.

\section{References}

1. Gandhi, R. T., Lynch, J. B., \& del Rio, C. (2020). Mild or Moderate Covid-19. New England Journal of Medicine., 383(18), 1757-1766
2. Wu, Z., \& McGoogan, J. M. (2020). Characteristics of and important lessons from the coronavirus disease 2019 (COVID19) outbreak in China: Summary of a report of 72314 cases from the Chinese center for disease control and prevention. JAMA, 323(13), 1239-1242

3. Silven, A. V., Petrus, A. H. J., Villalobos-Quesada, M., Dirikgil, E., Oerlemans, C. R., Landstra, C. P., Boosman, H., van Os, H. J. A., Blanker, M. H., Treskes, R. W., Bonten, T. N., Chavannes, N. H., Atsma, D. E., \& Teng, Y. K. O. (2020). Telemonitoring for patients with COVID-19: Recommendations for design and implementation. Journal of Medical Internet Research, 22(9), e20953. https://doi.org/10.2196/20953 Internet.

4. John, J., Council, L., Zallman, L., \& Blau, J. (2020). Developing an Intensive Community Covid-19 Management Strategy: Helping Our Patients Access Patient-Centered Care across a Continuum of Covid-19 Disease Needs. NEJM Catal Innov Care Deliv [Internet]. Retrieved May 27, 2020, from https://doi.org/ 10.1056/CAT.20.0181.

5. Zhou, F., Yu, T., Du, R., Fan, G., Liu, Y., Liu, Z., Xiang, Jie, Wang, Yeming, Song, Bin, Gu, Xiaoying, Guan, Lulu, Wei, Yuan, Li, Hui, Wu, Xudong, Xu, Jiuyang, Tu, Shengjin, Zhang, Yi., Chen, Hua, \& Cao, Bin. (2020). Clinical course and risk factors for mortality of adult inpatients with COVID19 in Wuhan, China: A retrospective cohort study. Lancet, 395(10229), 1054-1062

6. Kricke, G., Roemer, P., Barnard, C., Peipert, J., Henschen, B., Bierman, J., Blahnik, D., Grant, M., \& Linder, J. A. (2020). Rapid implementation of an outpatient covid-19 monitoring program. NEJM Catal Innov Care Deliv [Internet]. Retrieved June 16, 2020, from https://doi.org/10.1056/cat.20.0214.

7. Morgan, A. U., Balachandran, M., Do, D., Lam, D., Parambath, A., Chaiyachati, K. H., Bonalumi, N. M., Day, S. C., Lee, K. C., \& Asch, D. A. Remote Monitoring of Patients with Covid-19: Design, implementation, and outcomes of the first 3,000 patients in COVID Watch. NEJM Catal Innov Care Deliv [Internet]. https://doi.org/10.1056/CAT.20.0342. Retrieved December 23, 2021, from https://www.ncbi.nlm.nih.gov/pmc/articles/PMC73 $80701 /$.

8. Medina, M., Babiuch, C., Card, M., Gavrilescu, R., Zafirau, W., Boose, E., Giuliano, K., Kim, A., Jones, R., \& Boissy, A. (2020). Home monitoring for COVID-19. Cleveland Clinic Journal of Medicine [Internet]. Retrieved November 21, 2020, from http:// www.ccjm.org/content/early/2020/06/08/ccjm.87a.ccc028.abstr act.

9. Glasgow, R. E., Vogt, T. M., \& Boles, S. M. (1999). Evaluating the public health impact of health promotion interventions: The RE-AIM framework. American Journal of Public Health, 89(9), $1322-1327$

10. Department of Health and Human Services. (2020). Coronavirus update for Victoria. Retrieved December 1, 2020, from https:// www.dhhs.vic.gov.au/coronavirus-update-Victoria-01-Decem ber-2020.

11. Department Health and Human Services. (2020). Coronavirus update for Victoria. Retrieved November 15, 2020

12. Department Health and Human Services. (2020). Coronavirus update for Victoria. Retrieved August 5, 2020, from https://www. dhhs.vic.gov.au/coronavirus-update-victoria-5-august-2020.

13. Northern Health. (2020). Quality Account. Retrieved December 23, 2020, from https://www.nh.org.au/wp-content/uploads/2020/ 11/LR-NH-Quality-Account-2019-20.pdf.

14. Greenhalgh, T., Knight, M., A'Court, C., Buxton, M., \& Husain, L. (2020). Management of post-acute covid-19 in primary care. BMJ, 370, m3026

15. Tenforde, M., Kim, S., Lindsell, C., Billig Rose, E., Shapiro, N., $\&$ Files, D. (2020). Symptom duration and risk factors for delayed return to usual health among outpatients with COVID-19 in a 
multistate health care systems network-United States. MMWR Morbidity and Mortality Weekly Report, 69, 993-998

16. Eythorsson, E., Helgason, D., Ingvarsson, R. F., Bjornsson, H. K., Olafsdottir, L. B., Bjarnadottir, V., et al. (2020). Clinical spectrum of coronavirus disease 2019 in Iceland: Population based cohort study. BMJ, 371, m4529

17. Australian Government. (2020). COVID-19 Australia: Epidemiology report 30. Fortnightly reporting period ending 22 November 2020. COVID-19 National Incident Room Surveillance Team. Communicable Diseases Intelligence. Retrieved December 23, 2020, from https://doi.org/10.33321/cdi.2020.44.91
18. Hawkins, R. B., Charles, E. J., \& Mehaffey, J. H. (2020). Socioeconomic status and COVID-19-related cases and fatalities. Public Health, 189, 129-134

Publisher's Note Springer Nature remains neutral with regard to jurisdictional claims in published maps and institutional affiliations. 